\title{
Digital services for consumers
}

\author{
Jan Marco Leimeister $\cdot$ Hubert Österle $\cdot$ Steven Alter
}

Published online: 19 November 2014

(C) Institute of Information Management, University of St. Gallen 2014

This issue includes four papers that tackle important issues in the context of our special issue "Digital Services for Consumers". As we highlighted in our call for papers, mobile computing, high speed communication, internet of things, and a plethora of services available through several devices change the way we live. This is a huge potential but also a challenge for consumers as well as enterprises. We observe that this digitization of our everyday life has already led to a change in the preferences of the customers and this change will continue (Dellarocas 2013; Rechert et al. 2014; Reichhart et al. 2013). Successful services are often personalized, context adaptive, real-time, available anywhere, connected, and fun to use. The digital user has become powerful, since the competitor is just one click away. Thus, only perceivable usefulness, ease of use, and individual user-centricity of the service will bind the user to an offering. These innovative services have emerged first in the consumer world, making consumer markets for the first time the pacemakers of IT transformation. Furthermore, these consumer IT innovations change user expectations fundamentally, not only with respect to IT services, but to products and services in general. Everything as a service - when, how, and where each user wants it - becomes a paradigm for many successful new

J. M. Leimeister $(\bowtie)$

Resarch Center for IS Design (ITeG), Chair for Information Systems, University of Kassel, Pfannkuchstrasse 1, 34121 Kassel, Germany e-mail: JanMarco.Leimeister@unisg.ch

J. M. Leimeister $\cdot$ H. Österle

Institute of Information Management (IWI HSG), University of St. Gallen, Müller-Friedberg-Strasse 8, 9000 St. Gallen, Switzerland

H. Österle

e-mail: hubert.oesterle@unisg.ch

S. Alter

School of Management, University of San Francisco,

2130 Fulton Street, 94117 San Francisco, CA, USA

offerings. In this new Digital World, companies have much more problems understanding and anticipating customer demands since user behavior and preferences have changed dramatically. Most of the traditional approaches for designing and delivering products and services in this digital world seem outdated and incapable to address this fast changing environment of digital users (Leimeister et al. 2014). Consequently, we need to answer a vast amount of new questions: What services are useful, best adapted to each and every user? Which are fun to use? How to handle complexity of different offerings? What types of services have which benefit, when, how, and for whom? What services around product offerings are most beneficial for which purpose? Which services work best for digital natives and digital immigrants? What are underlying mechanisms for explaining successful design, use, and effects of digital services for consumers? Service innovations, new ways of service creation, orchestration, and consumption, using and creating vast amounts of openly available or user-generated data, offer great potential for improving everyday life of large parts of our society. Examples range from healthcare (with e.g., offerings such as patientslikeme.com) to new business models and ways of value creation and value capturing become critical, as a big opportunity, but also as a challenge for enterprises and other organizations. Electronic web services emerge for all areas of life, e.g., entertainment, communication, shopping, household, health, mobility, work, and finance.

These questions and opportunities are the outcomes of a transformation of our society towards a digital society. Historically, the advent of information technology (IT) in businesses led to a transformation from the industrial society into the information society, putting much emphasis on scalability of businesses through automization and standardization. Process orientation was the paradigm for efficiency gains, especially in back office environments. Today, the increasing ubiquity of IT and its penetration of our everyday life transforms the information society into the digital society, 
which means that many mechanisms from the internet become applicable to many other services (see Fig. 1) and that especially the power shift towards the digital user will also spill over to other areas. This digital society will lead to a new wave of business innovations. Especially start-ups will resemble value webs and disrupt traditional businesses and value chains. Such new offerings applying internet mechanisms to traditional markets such as transportation (e.g. UBER), housing (airbnb), and many more are able to conquer new markets - and these new entrepreneurs are able to address customer needs more effectively or even identify and satisfy new needs in the digital age.

In digital societies, companies must understand that the digital customers and their preferences are key and at the center stage for developing innovative solutions. The principles of user-, use-, utility-centricity (Brenner et al. 2014) are crucial to success, as they can support companies and entrepreneurs in innovating and calibrating their offerings. The principle of user-centricity is quite simple. The digital users and their needs should be the main interest of a company, and always serve as the basis for developing and offering new products and services - services from people for people. The concept of use-centricity addresses the importance of usability and user experience for market success. Apple serves as a perfect example here, since it set hallmarks in usability for everyone - no matter how old or how interested in technology - everyone is almost intuitively able to use Apple's innovations and very often with joy of use. Furthermore, successful businesses are very good at understanding which value they are able to provide for whom. This idea is captured in the principle of utility-centricity, which highlights the importance of analyzing the different kinds of value which are generated for all the different stakeholders. A good example for a utilitycentric design of solutions are captchas. Besides being a security feature, captchas often fulfill at least one other purpose. In some solutions, the users are asked to type in two words they need to read from two screenshots. Here, usually only one word serves as the actual test. The answers given to the second word are often used to, e.g., improve algorithms for optical character recognition. This clever design from a utility point of view offers captcha providers further options for additional utility and monetization.

A suitable framework for both researchers and practitioners to address the challenges of digitization is the St. Galler House of Digital Business (Fig. 2). It uses the core structure known from the traditional Business Engineering model (Österle and Winter 2000) - consisting of the layers of business strategy, processes, and IT - represented by the columns managerial functions and an outcome perspective - products and services. In addition, the user-, use-, utility-centricity resembles the roof of the house, and data serves as glue tying all parts together. The first extension is necessary because the digitization increasingly influences how we work. Consequently, executives need to perform additional management tasks, some of which require new skills. The emphasis on the outcome perspective in form of a separate column is required because IT more often becomes an essential part of the products and services offered in the digital age, leading to new questions concerning the development and combination of products and services. The user-, use-, utility-centricity as roof represents the overarching character of these design principles. They define how every component in the St. Galler House of Digital Business has to
Fig. 1 The Path to the Digital Society (Leimeister et al. 2014)

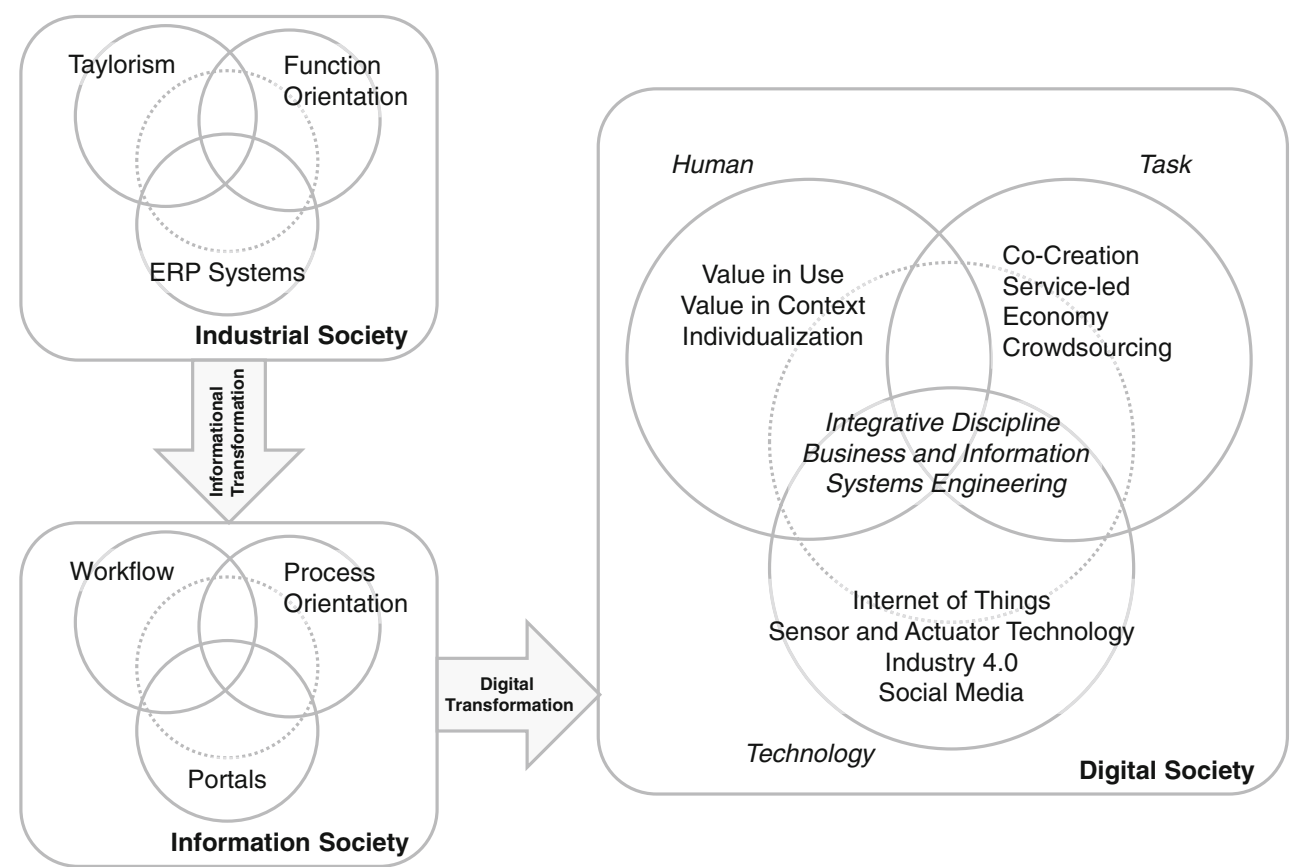




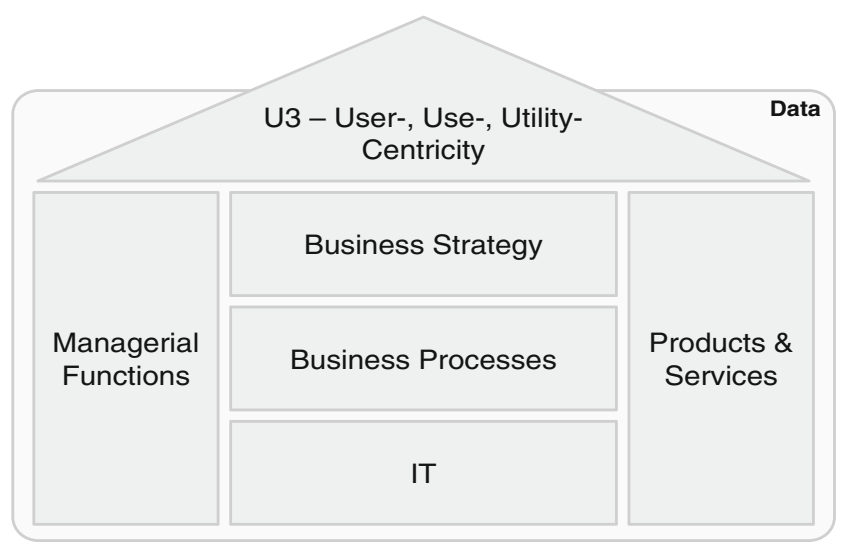

Fig. 2 St. Galler House of Digital Business (Leimeister et al. 2014)

be interpreted, putting the digital customers and their preferences and needs in the core of every activity. The digital customers, both internal and external, expect personalized products and services customized to their needs and current situation. Therefore, specific business solutions need to be developed, namely solutions that address digital users' needs and consider economic considerations in order to generate the greatest benefit possible for all involved parties. Data accounts for the increasing importance of Big Data for companies. Leading companies such as Google and Amazon have mastered drawing conclusions about needs and future behavior of the customers based on existing data. These conclusions then, for example, serve as an input for the development of new products and services, or the definition of new business strategies and processes.

All papers of this special issue contribute to the understanding of the relevance of user-, use-, utility-centricity, which we regard as a core challenge for companies in various business domains.

The first paper of this special issue "Converting freemium customers from free to premium - The role of perceived premium fit in the case of music as a service" by Thomas M. Wagner, Alexander Benlian, and Thomas Hess focuses on online-based music services, or more specifically, on music service providers - such as Spotify, Deezer, or Pandora. It is nowadays possible to listen to almost every imaginable song with a free and hence costless account on one of the online music providers. However, this content comes at a cost for the providers, which is why they have to cross-finance such offers somehow. Embedded advertisement is only one of the possibilities whilst the freemium approach is another one which is becoming more popular - especially with respect to establishing a user-friendly cross-finance solution. Nowadays, we find various internet services based on the freemium concept - such as LinkedIn, Dropbox, or Skype. Freemium services are free of charge; however, they do not include all functionalities. Consumers can access richer functionality for a subscription fee (Kumar 2014). In the context of freemium related limitations of functionalities, the study by Wagner et al. "aims to measure whether a free service's limitations impact the evaluation of the freemium and premium versions" and hence affect the conversion rate from free to premium customers. The underlying research model extends the Dual Mediation Hypothesis by the similarity between the "free and premium versions' functionality" (perceived premium fit) and the "perceived value of the premium version compared to its price" (perceived price value) and uses the Elaboration Likelihood Model on a dataset of 317 music service users. The results of the study show that the functional gap between the free and premium version of a service is an important factor for the users' evaluation of a free version, whereas a high premium fit and hence small functional gap leads to a positive attitude towards the free version. This effect may also improve the conversion rate as free versionbased advertising effects are "stronger for users who believe they experienced only small functional differences". Based on the generated insights, the authors recommend maximizing the premium fit in order to increase "the probability of users' conversion and would reduce a freemium service's costs." Further, providers are suggested to offer time-limited trial periods in order to avoid the risk of free-riding.

Ensuing from the customer-centric perspective, the second paper of this special issue authored by Hua Dai and Al F. Salam ("Does Service Convenience Matter? An Empirical Assessment Of Service Quality, Service Convenience And Exchange Relationship in Electronic Mediated Environment") analyses how service convenience in conjunction with online service content quality affects long-term relationships between customers and online service providers in the electronic mediated environment (EME). EME services include, for instance, online entertainment services or electronic banking services. The authors argue that service convenience is an important factor that affects consumer behavior in EMEs and further emphasize "the customer-centric role as most important business strategy to serve and create value for customers effectively." Following this argumentation, the authors develop a research model which attributes "service convenience" on "service delivery quality" as well as "service content quality." The model also suggests a positive relationship between service convenience and the "perceived value of service" as well as a "consumer's commitment towards a service" in EME. The authors test their model using a dataset consisting of 772 respondents and find that service convenience is positively influenced by service delivery quality as well as service content quality. Meanwhile, perceived service convenience positively influences perceived value as well as the commitment towards a service - which both enhance word-of-mouth communication as well as loyalty as shown by the results. Based on these insights, the authors recommend laying emphasis on issues that affect service delivery quality i.e., accessibility, timeliness, and reliability - and service content quality - i.e., visual appearance, flexibility, and navigation - when designing online provider websites. Most 
notably, this study stresses the necessity of considering digital users' needs and interests as the basis for developing and offering EME services as outlined in the St. Galler House of Digital Business.

Compared to the two first papers, the third and the fourth paper focus on services related to social networking sites. In the third paper of this special issue ("Influencing Customer's Purchase Intentions through Firm Participation in Online Consumer Communities"), Leslie J. Albert, Nitin Aggarwal, and Timothy R. Hill argue that social networking sites and online consumer communities, in particular, can be remarkably essential marketing tools. Online consumer communities provide consumers a platform for discussing products or services with other consumers or even with firm representatives. We can clearly observe that companies are increasingly getting involved in such social communities for different reasons as shown by the authors: For instance, for increasing brand awareness, for improving a customer's image of a firm, or for enhancing customer relationships. The authors seize on this fact and examine how a service provider's participation in online user communities can affect customers' purchase intention. They focus on third-party-hosted sites, which are not controlled by the associated firms, and analyze the activity of three hotel chains within a social consumer community dedicated to travelling. The authors find that both "perceived post frequency" of service providers as well as the value of these posts perceived by consumers positively influence consumers' hotel choice preferences. Based on these results, the authors recommend service providers to increase the frequency of their participation in online consumer communities. Further, the authors suggest that the post value as perceived by the consumers is due to the type of posts: For instance, the post value of the provider focusing on consumers' concerns and requests is appraised much higher than the post value of the provider focusing primarily on promotional announcements.

The fourth study is authored by Aikaterini Manthiou, Liang Tang, and Robert Bosselman ("Reason and reaction: The dual route of the decision-making process on Facebook fan pages") and analyses the dual routes of consumers' decision-making processes on behavioral change toward purchase on Facebook fan pages. Nowadays, a Facebook fan page is almost inevitable for firms, especially for B2C companies. Using the Prototype Willingness Model, the authors develop a research model that mainly suggests that "behavioral change toward purchase" is positively influenced by "behavioral intention toward fan page" (i.e., using or recommending a provider's Facebook page) as well as the "behavioral willingness toward fan page" (i.e., further usage of a provider's Facebook page). The latter constructs are, in turn, influenced by other factors such as attitude towards the fan page (determined, for instance, by fan pages design characteristics), subjective norms, or prototype image (i.e., "a person's mental image of the type of people who are interested in a certain behavior"). The results report positive relationships between these constructs and confirm that the dual routes of decision making (reasoned path and reaction path) occur simultaneously. Based on these insights, the authors derive various design recommendations that positively influence the important factor of "attitude towards the fan page" such as the use of a fan page wall and online chat features that support communication between a company and consumers. Further, the authors recommend regularly updating content and responding to consumers' posts as well as providing unique, interesting, and creative fan page designs. In doing so, users can experience the benefits of the user-centric fan page design.

The four articles of this issue altogether underline the importance as well as the applicability of the St. Galler House of Digital Business with respect to digital services for consumers service providers are encouraged to design services based on customers' needs and preferences, thereby perpetually considering customer's exploitation of digital offerings. This alignment promotes the probability of consumers using these services. The usage will lead to a clear benefit both for the customers - i.e., by receiving services tailored to individual preferences - as well as for the service providers that benefit from loyal customers.

Our thanks go to the highly competent and supportive team of reviewers. Without hard working authors and reviewers we would have never been able to produce such a special issue. We hope you perceive it as worthwhile and enjoy reading and using it.

\section{References}

Brenner, W., Karagiannis, D., Kolbe, L., Krüger, J., Lamberti, H.-J., Leifer, L., et al. (2014). User, use \& utility research: the digital user as new design perspective in business \& information systems engineering. Business \& Information Systems Engineering (BISE), 6(1), $55-61$.

Dellarocas, C. (2013). The digitization of word of mouth: promise and challenges of online feedback mechanisms. Management Science, 40(10), 1407-1424.

Kumar, V. (2014). Making "Freemium” Work. Harvard Business Review, May 2014.

Leimeister, J. M., Winter, R., Brenner, W. \& Jung, R. (2014). Research Program "Digital Business \& Transformation HSG". Working Paper Services of University of St. Gallen's Institute of Information Management. Available at SSRN: http://ssrn.com/abstract= 2501345.

Österle, H., \& Winter, R. (2000). Business engineering. In H. Österle \& R. Winter (Eds.), Business engineering - Auf dem Weg zum Unternehmen des Informationszeitalters. (pp. 3-20). Berlin: Springer.

Rechert, K., Suchodoletz, D., Valizada, I., Latocha, J., Cardenas, T. J., \& Kulzhabayev, A. (2014). Take care of your belongings today securing accessibility to complex electronic business processes. Electronic Markets, 24(2), 125-134.

Reichhart, P., Pescher, C., \& Spann, M. (2013). A comparison of the effectiveness of e-mail coupons and mobile text message coupons for digital products. Electronic Markets, 23(3), 217-225. 